\title{
ESTIMATIVA DO TEMPO NECESSÁRIO PARA ALCANÇAR O PERÍODO PRECONIZADO DO ALEITAMENTO MATERNO
}

\section{ESTIMATION OF TIME NECESSARY TO ACHIEVE THE PREVIOUSLY PERIOD BREASTFEEDING}

\author{
Gheisa Helen Signolfi-Lopes ${ }^{1}$, Esmael Lopes dos Santos ${ }^{2 *}$
}

\begin{abstract}
${ }^{1}$ Enfermeira, Pós graduada em Aleitamento Materno pela UNIFIL, pós graduada em saúde coletiva e da família pela UEL, é Enfermeira da linha de cuidado materno infantil da Unimed, Londrina - Paraná,

Brasil. Biólogo, Agrônomo, Mestre e Doutor pela UEL, Professor do curso de Agronomia da FAG
\end{abstract}

*Autor correspondente: elsantos@fag.edu.br http://orcid.org/0000-0001-8206-4881

DOI: $10.35984 /$ fjh.v2i2.200

\section{RESUMO}

O objetivo do trabalho foi buscar na literatura tempo de aleitamento materno (AM) e de aleitamento materno exclusivo (AME) para estimar o tempo necessário para alcançar 0 período preconizado. Para alcançar o objetivo do trabalho, foram realizadas revisões bibliográficas para obter dados do tempo AM e de AME. Os dados obtidos foram submetidos a análise de regressão, e, avaliados quanto ao grau de significância por meio do software Sigmaplot 9.0. Foram encontrados na literatura sete anos (1975, 1982, 1989, 1996, 1999, 2006 e 2013) com valores da mediana do AM. Para o AME foram encontrados na literatura somente quatro anos (1999, 2000, 2008 e 2013) com valores de mediana. A estimativa encontrada é que a mediana do AM se classifica em 2019 como bom (20 a 22 meses), e que somente em 2022 a mediana chegará em 24 meses, o que classificaria como muito bom (>23 meses) e preconizado pelo ministério da saúde. A mediana do AME é classificada em 2019 como ruim (3,6 a 4,5 meses), e que somente em 2041 a mediana chegará em seis meses conforme o preconizado pelo ministério da saúde, o que classificaria como muito bom $(>5,5$ meses).

Palavras-chave: aleitamento materno, lactente, desmame precoce.

\section{ABSTRACT}

The objective of the study was to search the literature for breastfeeding time (BF) and exclusive breastfeeding time (BF) to estimate the time needed to reach the recommended period. To achieve the objective of the work, bibliographic reviews were carried out to obtain data on the $A M$ and AME time. The data obtained were submitted to regression analysis, and evaluated for the degree of significance using the Sigmaplot 9.0 software. Seven years $(1975,1982$, 1989, 1996, 1999, 2006 and 2013) were found in the literature with values for the median BF. For EBF, only four years (1999, 2000, 2008 and 2013) were found in the literature with median values. The estimate is that the median MA is classified as good (20 to 22 months) in 2019 and that in 2022 the median will reach 24 months, which would be very good (> 23 months) and recommended by the WHO. The median EBF is classified as bad in 2019 (3.6 to 4.5 months), and that in 2041 the median will reach six months as recommended by the WHO, which would rank as very good (> 5.5 months ).

Keywords: breastfeeding, infant, early weaning. 


\section{INTRODUÇÃO}

O leite materno é o alimento mais completo para a nutrição do recém-nascido nos primeiros seis meses de vida. $\mathrm{O}$ aleitamento materno exclusivo diminui o índice de mortalidade infantil por patologias comuns da infância, como diarréia e pneumonia, e ajuda na recuperação mais rápida da mulher no pós-parto. No entanto, diversos motivos têm levado as nutrizes a optarem pelo desmame precoce dos seus filhos, buscando caminhos alternativos para a nutrição dos mesmos (PEREIRA E REINALDO, 2018).

O desmame precoce é considerado antes dos seis meses de vida independente da causa, sendo caracterizado de forma tranquila ou não dependendo das dificuldades ou vivências encontradas (GIUGLIANI et al., 2008). A Organização Mundial de Saúde (OMS) recomenda o Aleitamento Materno Exclusivo (AME) até o sexto mês de vida e a continuidade do aleitamento materno até o segundo ano de vida ou mais (BRASIL, 2009). As principais consequências do desmame de crianças menores de seis meses de idade, considerado precoce, são: aumento da mortalidade infantil; aparecimento de doenças alérgicas, cânceres, obesidade, diabetes, deficiência no desenvolvimento cognitivo e emocional da criança, anemia ferropriva e doenças cardiovasculares (BARROS, 2009).

Segundo Moraes et al., (2014), os motivos que levam ao desmame precoce variaram de acordo com a cultura e contexto histórico de cada lugar. No Brasil, a partir de 1980, a importância do aleitamento materno para o bem do lactente, da mãe e da sociedade como um todo é levada ao público por meio de campanhas publicitárias. Entretanto, apesar de tais esforços, o desmame precoce no Brasil ainda era expressivo. Muitas mulheres não conseguiam amamentar por produzir menor quantidade de leite ou pelo fato do mesmo secar facilmente. Sem saber lidar com esse tipo de problema, os higienistas criaram o conceito de leite fraco, idéia essa que parece desmotivar até hoje muitas mães de amamentar. Apesar disso, mesmo com os problemas criados devido ao falso conceito de leite fraco, tendo em vista que atualmente é comprovado que as disfunções lactôgênicas são raras (BOCCOLINI, et al., 2017). Estudos realizados por Rocci e Fernandes (2014), não comprovaram correlação entre o tempo de aleitamento materno exclusivo e as vaiáveis: estado civil, idade materna, peso do recém-nascido e orientações recebidas. Os autores identificaram que as mães que apresentaram dificuldades na amamentação tiveram, em média, menor tempo de aleitamento exclusivo, ou seja, a dificuldade na amamentação influenciou o tempo de aleitamento materno exclusivo. Vale ressaltar que as mães que citaram dificuldade em amamentar já na pré-alta, apresentaram aos 60 dias um percentual de desmame significativamente maior que aquelas que não tiveram dificuldades. No entanto, os estudos de Pereira e Reinaldo (2018) apontaram com maior frequência do desmame precoce o uso de chupeta, trabalho materno, dificuldade em amamentar, baixa renda familiar e intercorrências mamárias.

Estimativas de variáveis que não há resultados ressentes ou que se busca prever regularidade e tempo para alcançar determinado valor, são utilizadas com frequência. As estimativas podem ser realizadas, submetendo determinada sequência de dados à análise de regressão e utilizando equações calculadas para realizar as estimativas. Neste contexto, Nejar et al., (2004), utilizou a metodologia de Drewett et al., (1989), para estimar o consumo de leite materno, por ter baixo custo, ser de fácil aplicação e não exigir tecnologia. Por este método, estima-se o volume de leite materno através de regressão linear múltipla. 
Desta forma, o presente trabalho tem como objetivo buscar na literatura tempo de aleitamento materno (AM) e de aleitamento materno exclusivo (AME) para estimar o tempo necessário para alcançar o período preconizado.

\section{METODOLOGIA}

Para alcançar o objetivo do trabalho, foram realizadas revisões bibliográficas para obter dados do tempo de aleitamento materno (AM) e de aleitamento materno exclusivo (AME). Após o levantamento, seguindo metodologia de Nejar et al., (2004) e Drewett et al., (1989), os dados obtidos foram submetidos a análise de regressão referentes aos seguintes modelos:
1) $\mathrm{AM}$ ou $\mathrm{AME}=\mathrm{e}^{\beta 0+\beta 1 \mathrm{CAP}^{-1}}$ (Exponencial)
2) $\mathrm{AM}$ ou $\mathrm{AME}=\mathrm{e}^{\beta 0+\beta 1 \text { InCAP }}$ (Potência)
3) $\mathrm{AM}$ ou $\mathrm{AME}=\beta 0+\beta 1 \mathrm{CAP}$ (Polinômio)

Considerando o critério de maior $\mathrm{R}^{2}$, o modelo que obteve o melhor desempenho ao se estimar tanto o $\mathrm{AM}$ e $\mathrm{AME}$ quanto aos dados encontrados na literatura foi à equação da regressão do modelo exponencial (1). Os resultados das análises de regressão foram avaliadas quanto ao grau de significância por meio do software Sigmaplot 9.0. Os resultados obtidos de AM foram classificados como: muito ruim para a mediana menor que 17 meses; ruim, de 18 a 20 meses; bom, de 21 a 22 meses e, por fim, muito bom, maior que 24 meses, seguindo os parâmetros OMS citados por Toryiama et al., (2017). Seguindo os parâmetros utilizados para o AM, foram sugeridos parâmetros para a classificação de AME, nas seguintes categorias: muito ruim para a mediana menor que 3,5 meses; ruim, de 3,6 a 4,5 meses; bom, de 4,6 a 5,5 meses e, por fim, muito bom, maior que 5,6 meses.

\section{RESULTADOS E DISCUSSÃO}

$\mathrm{Na}$ tabela 1, estão dispostos os artigos analisados para a caracterização e levantamento dos dados de aleitamento materno (AM) para a realização da estimativa do período necessário para alcançar os 24 meses de amamentação preconizados pelo ministério da saúde (BRASIL, 2009). No ano de 1975 a mediana da amamentação era de 2,5 meses e na década de 80 é notável o considerável aumento no tempo de amamentação de 4,0 meses em 1982 e 5,5 meses em 1989. Segundo Santo (2010), pesquisas no âmbito nacional tem demonstrado que, desde a implantação do programa nacional de incentivo ao aleitamento materno, no início da década de 1980, os índices de aleitamento no país vêm aumentando.

Tabela 1. Caracterização do levantamento dos dados de aleitamento materno (AM) para estimativa do período necessário para alcançar os 24 meses preconizados pela Organização Mundial da Saúde. Londrina, PR., 2019.

\begin{tabular}{ccc}
\hline Ano & Aleitamento (meses) & Autor \\
\hline 1975 & 2,5 & SANTO, 2010 \\
1982 & 4,0 & SANTO, 2010 \\
1989 & 5,5 & SANTO, 2010 \\
1996 & 7,0 & SANTO, 2010 \\
1999 & 9,9 & SANTO, 2010 \\
2006 & 14,0 & BRASIL, 2009 \\
2013 & 21,3 & BUCOLLINI, 2011 \\
\hline
\end{tabular}


Na década de 90 houve aumento na mediana da amamentação de 7,0 meses de 1996 e 9,9 meses em 1999. Segundo Santo (2010) em 1991 foi lançada a iniciativa hospital amigo da criança (IHAC), que buscou resgatar direitos da mulher de aprender a pratica de amamentação, assim como em 1992 foi aprovada a norma brasileira de comercialização de alimentos para lactentes (NBCAL). No ano de 1995 o Ministério da Saúde lançou o projeto de redução da mortalidade infantil, com o objetivo de reduzir óbitos infantis e a melhoria da situação de saúde das crianças mediante ações do aleitamento. No ano de 1998, o ministério da saúde criou a rede nacional de banco de leite humano (BLH) e a partir de 1999 o Ministério da Saúde passou a coordenar a semana nacional de amamentação, comemorado anualmente nos dias 1 a 7 de agosto. No ano de 2006 houve um aumento de 14 meses na mediana da amamentação, assim como no ano de 2013 o aumento foi de 21,3 meses. Conforme Santo (2010), em 2000 países das nações unidas, entre os quais o Brasil, firmaram objetivos na redução da mortalidade de crianças menores de cinco anos de 1990 a 2015.

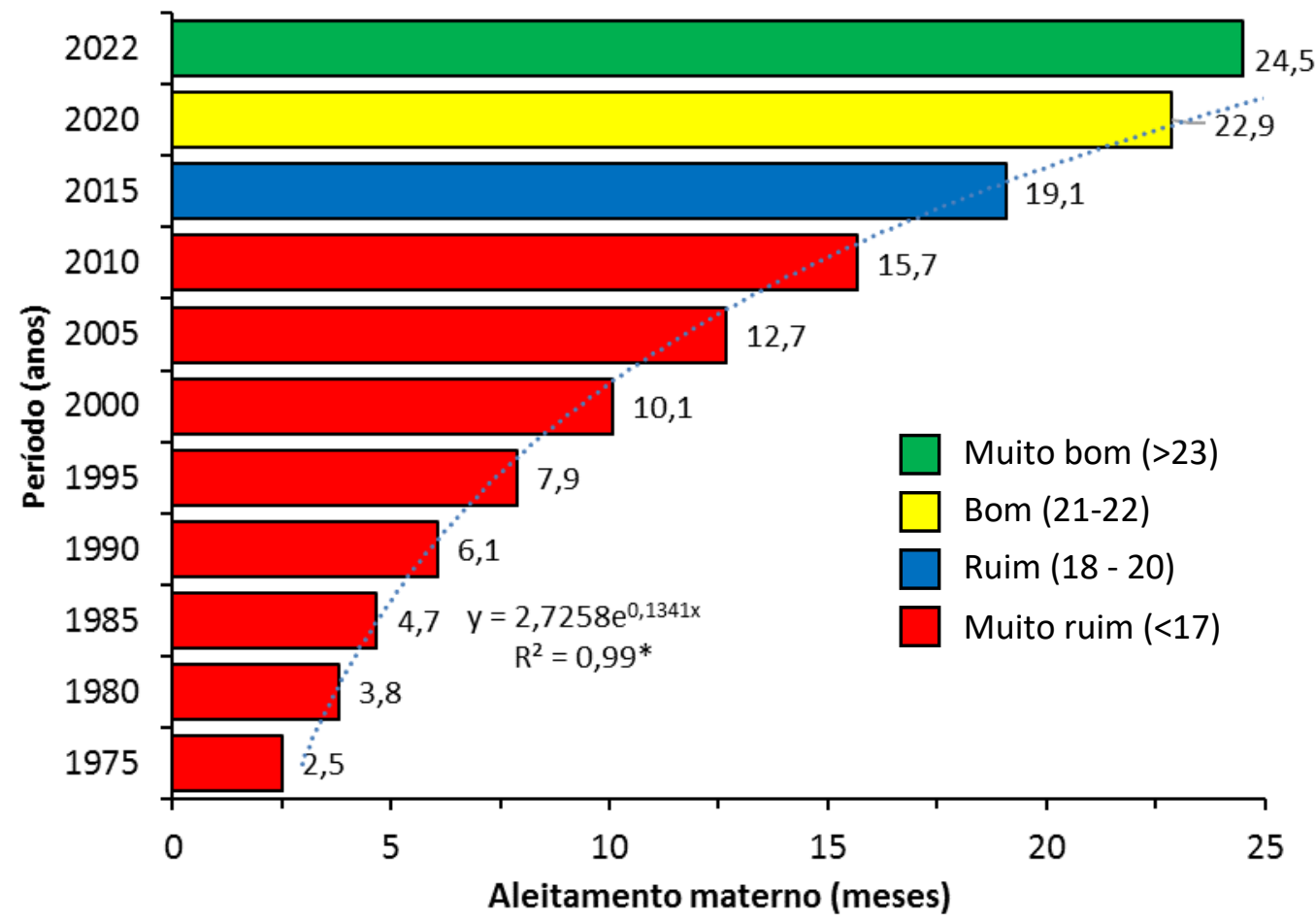

Figura 1. Duração mediana do aleitamento materno (valores observados: 1975, 1982, 1989, 1996, 1999, 2006 e 2013) os outros valores foram calculados conforme equação gerada pelos valores observados. Londrina, PR., 2019. ( ${ }^{*}$ Significativo a $\left.1 \%\right)$.

De acordo com a mediana do aleitamento materno dos anos analisados (Figura 1) e a partir da equação gerada, houve uma classificação conforme os parâmetros da OMS citados por Toryiama et al., (2017). Neste caso, entre os anos de 1975 e 2010 a classificação foi de muito ruim ( $<17$ meses de aleitamento materno). O ano de 2015 foi classificado como ruim ( $18-20$ meses de aleitamento materno). Na estimativa, o ano de 2020 será bom (20 - 22 meses de aleitamento materno), seguida por 2022 que será muito bom (>23 meses de aleitamento materno), e neste caso chegaria aos 24 meses, que segundo o caderno de atenção básica (CAB 23), a OMS, endossada pelo Ministério da Saúde do Brasil (BRASIL, 2009), recomenda aleitamento materno por 2 anos ou mais. 
$\mathrm{Na}$ tabela 2, estão dispostos os artigos analisados para a caracterização e levantamento dos dados de aleitamento materno exclusivo (AME) para a estimativa do período necessário para alcançar os seis meses de amamentação exclusiva preconizada pelo ministério da saúde (BRASIL, 2009).

Tabela 2. Caracterização no levantamento dos dados do aleitamento materno exclusivo (AME) para estimativa do período necessário para alcançar os seis meses preconizados pelas Organização Mundial da Saúde. Londrina, PR., 2019.

\begin{tabular}{ccc}
\hline Ano & Aleitamento (meses) & Autor \\
\hline 1999 & 0,78 & Brasil, 2009 \\
2000 & 0,93 & Brasil, 2009 \\
2008 & 1,8 & Brasil, 2009 \\
2013 & 2,4 & BUCOLLINI, 2011 \\
\hline
\end{tabular}

No ano de 1999, a amamentação exclusiva acontecia por 0,78 meses e apenas no ano de 2008 foi observado um discreto aumento para 1,8 meses. Segundo Salustiano et al, (2011), o AME definido pela OMS como a oferta apenas de leite, sem mesmo água ou chá, permitindo-se apenas gotas de vitaminas ou medicamentos - é preconizado até o sexto mês de vida das crianças. Quando estas crianças recebem outros tipos de alimentos diferentes do leite materno antes de completar 180 dias de vida, pode se atribuir que houve início do processo de desmame precoce.

Na figura 2, está disposta a duração mediana do aleitamento materno exclusivo (AME) nos anos de 1999, 2000, 2008 e 2013 e a estimativa até o ano de 2041.

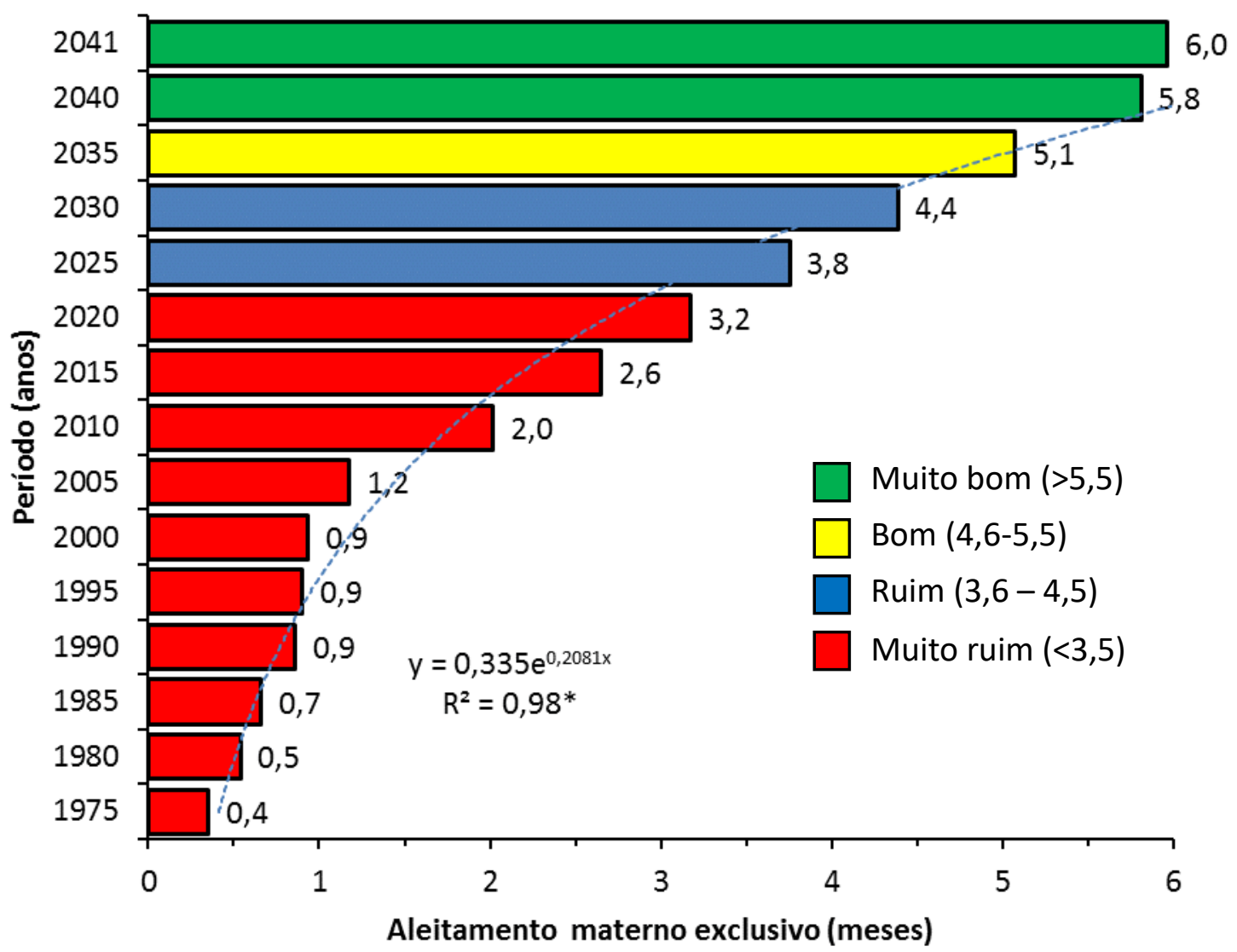

Figura 2. Duração mediana do aleitamento materno exclusivo (valores observados: 1999, 2000, $2008,2013)$ e valores calculados conforme equação gerada pelos valores observados. Londrina, PR., 2019. ( ${ }^{\star}$ Significativo a $\left.1 \%\right)$. 
De acordo com Giugliani (2008), qualquer esforço no sentido de aumentar as taxas de AME deve levar em consideração os fatores que influem negativamente nesta prática. Os principais obstáculos a esta prática podem ser assim agrupados: falta de conhecimento e conscientização da população em geral, dos profissionais de saúde e dos gestores; condutas inapropriadas e pouca qualificação dos profissionais de saúde; aspectos culturais; falta de confiança/baixa autoestima da mãe; falta de apoio e suporte familiar e comunitário; trabalho da mulher; e promoção inapropriada de substitutos do leite materno.

Segundo Salustiano et al, (2011), no Brasil é baixo o número de mães que amamentam exclusivamente seus filhos até o sexto mês e complementado até os dois anos ou mais, como recomenda a OMS. O AME por pelo menos quatro meses tem sido raramente praticado. Fatores como a inserção da mulher no mercado de trabalho, durante o processo de industrialização e urbanização, contribuíram para o aumento das práticas de desmame, principalmente devido à ausência de garantias trabalhistas e medidas de proteção. Além disso, há descrição de que o emprego de chupetas, bem como baixos níveis de educação formal materna podem promover o desmame precoce (BOCCOLINI, et al., 2017).

\section{CONSIDERAÇÕES FINAIS}

Foram encontrados na literatura sete anos (1975, 1982, 1989, 1996, 1999, 2006 e 2013) com valores da mediana do AM. Para o AME foram encontrados na literatura somente quatro anos (1999, 2000, 2008 e 2013) com valores de mediana. A estimativa encontrada é que a mediana do AM se classifica em 2019 como bom (20 a 22 meses), e que somente em 2022 a mediana chegará em 24 meses o que classificaria como muito bom (>23 meses) e preconizado pelo ministério da saúde. A mediana do AME é classificada em 2019 como ruim (3,6 a 4,5 meses), e que somente em 2041 a mediana chegará a seis meses conforme o preconizado pelo ministério da saúde, 0 que classificaria como muito bom ( $>5,5$ meses).

\section{REFERÊNCIAS}

BARROS, K.M.; BRITO, J.A.; VIANA, M.F.A.; VERAS, J.M.M.F. Desmame precoce:

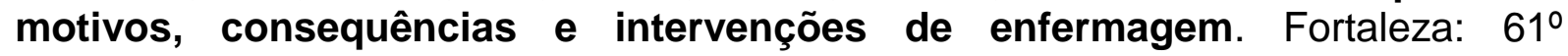
Congresso Brasileiro de Enfermagem. 2009. Disponível em: http://www.abeneventos.com.br/anais_61cben/files/01232.pdf. Acesso em: 13 de maio de 2019.

BOCCOLINI, C. S.; BOCCOLINI, P. M. M.; MONTEIRO, F.R.; VENÃNCIO, S.I.; GUGLIANI, E.R.J. Tendência de indicadores do aleitamento materno no Brasil em três décadas. Revista de Saúde Pública, v. 45, n. 1, p. 69-78, 2017. http://dx.doi.org/10.11606/s1518-8787.2017051000029

BRASIL, Departamento de Ações Programáticas e Estratégicas, Secretaria de Atenção à Saúde, Ministério da Saúde. II Pesquisa de Prevalência de Aleitamento Materno nas Capitais Brasileiras e Distrito Federal. Brasília, 2009. Disponível em http://bvsms.saude.gov.br/bvs/publicacoes/pesquisa prevalencia aleitamento mater no.pdf (acessado em 13/Mai/2019). 
DREWETT, R.F.; WOOLRIDGE, M.W.; JACKSON, D. A.; IMONG, S.M.; MANGKLABRUKS, A.; WONGSAWASDII, L.; et al. Relationships between nursing patterns, supplementary food intake and breast-milk intake in a rural Thai population. Early Hum Dev, V.20, P. 13-23, 1989. https://doi.org/10.1016/0378-3782(89)90069-8

GIUGLIANI, E.R.; ESPÍRITO SANTO, L.C.; OLIVEIRA, L.D.; AERTS, D. Intake of water, herbal teas and non-breast milks during the first month of life: associated factors and impact on breastfeeding duration. Early Hum Dev. v.84, p.305-10, 2008. DOI: 10.1016 / j.earlhumdev.2007.08.001

MORAES, J.T.; OLIVEIRA, V.A.C.; ALVIN, E.A.B.; CABRAL, A.A.; DIAS, J.B. A percepção da nutriz frente aos fatores que levam ao desmame precoce em uma unidade básica de saúde de Divinópolis/MG. R. Enferm. Cent. O. Min. v.4(1), p. 971982, 2014. DOI: https://doi.org/10.19175/recom.v0i0.446

NEJAR, F.F.; SEGALL-CORRÊA, A.M.; RÉA, M.F.; VIANNA, R.P.T.; PANIGASSI, G. Padrões de aleitamento materno e adequação energética. Cad Saúde Pública, v.20, p.64-71, 2004. https://doi.org/10.1590/S0102-311X2004000100020.

PEREIRA, N.N.B.; REINALDO, A.M.S. Não adesão ao aleitamento materno exclusivo até os seis meses de vida no brasil: uma revisão integrativa. Revista de APS. v. 21 n. 2, 2018. https://doi.org/10.34019/1809-8363.2018.v21.16281

ROCCI, E.; FERNANDES, R. A. Q. Dificuldades no aleitamento materno e influência no desmame precoce. Rev. bras. enferm. v.67(1), p. 22-27, 2014. https://doi.org/10.5935/0034-7167.20140002

SALUSTIANO, L.P.Q.; DINIZ, A.L.D.; ABDALLAH, V.O.S; PINTO, R.M.C. Fatores associados à duração do aleitamento materno em crianças menores de seis meses. Ver. Bras.Ginecol. Obstet, v. 34, p. 28-33, 2017.https://doi.org/10.1590/S0100$\underline{72032012000100006}$

SANTO, L. C. E. Políticas públicas em aleitamento materno. In: Carvalho MR, Tavares LAM, editores. Amamentação: bases científicas. 3. ed. Rio de Janeiro: Guanabara Koogan; p. 277-285, 2010.

TORYIAMA, Á.T.M.; FUGIMORI, E.; PALOMBO, C.N.T.; DUARTE, L.S.; BORGES, A.L.V.; CHOFAKIAN, C.B.N. Aleitamento materno: o que mudou após uma década? Rev. Latino-Am. de Enfermagem. 25:E2941, 2017. https://doi.org/10.1590/1518$\underline{8345.1858 .2941}$ 